\title{
Expression profiles of inhibitor of growth protein 2 in normal and cancer tissues: An immunohistochemical screening analysis
}

\author{
SHUANG ZHAO, XUE-FENG YANG, WEN-FENG GOU, HANG LU, \\ HUA LI, ZHI-TU ZHU, HONG-ZHI SUN and HUA-CHUAN ZHENG
}

Cancer Center and Key Laboratory of Brain and Spinal Cord Injury of Liaoning Province, The First Affiliated Hospital of Liaoning Medical University, Jinzhou, Liaoning 121001, P.R. China

Received November 23, 2014; Accepted August 25, 2015

DOI: $10.3892 / \mathrm{mmr} .2015 .4723$

\begin{abstract}
Inhibitor of growth protein 2 (ING2) has an important role in the regulation of chromatin remodeling, cell proliferation, cell-cycle arrest, senescence and apoptosis. The present study performed an immunohistochemical analysis for expression profiling of ING2 protein in an array of tissues comprising normal mouse and human tissues, as well as human hepatocellular $(n=62)$, renal clear cell $(n=62)$, pancreatic $(n=62)$, esophageal squamous cell $(n=45)$, cervical squamous cell $(n=31)$, breast $(n=144)$, gastric $(n=196)$, colorectal $(n=96)$, ovarian $(n=208)$, endometrial $(n=96)$ and lung $(n=192)$ carcinoma tissues. In mouse tissues, ING2 was detected in the nuclei and cytoplasm of the glandular epithelium of breast, hepatocytes, intestine, bronchium and alveoli, as well as the squamous epithelium of skin and glomeruli, and in myocardial cells, while it was located in the cytoplasm of renal tubules and striated muscle cells. ING2 protein was scattered in the brain and spleen. In human tissues, ING2 protein was principally distributed in the cytoplasm, while in it was present in the cytoplasm and nuclei in the stomach, intestine, cervix, endometrium trachea, breast and pancreas. The nuclear location of ING2 in the stomach was more prominent than that in the cytoplasm. High ING2 immunoreactivity was detected in the tongue, stomach, skin, pancreas, cervix and breast, whereas weakly in the brain stem, thymus, thyroid, lung, striated muscle, testis, bladder and ovary. In total, 617 out of 1,194 of the tested cancer tissues $(51.7 \%)$ were ING2-positive. In most cases, ING2 expression was found to be restricted to the cytoplasm of all cancer tissues, while in certain cancer types, including renal clear cell, ovarian and colorectal carci-
\end{abstract}

Correspondence to: Professor Hua-Chuan Zheng, Cancer Center and Key Laboratory of Brain and Spinal Cord Injury of Liaoning Province, The First Affiliated Hospital of Liaoning Medical University, 2 Five Sections, Renmin Street, Jinzhou, Liaoning 121001, P.R. China

E-mail: zheng_huachuan@ hotmail.com

Key words: inhibitor of growth protein 2, immunohistochemistry, expression profile, mouse, human, cancer noma, it was occasionally present in the nuclei. Among the cancer tissues examined, ING2 was most frequently expressed in breast cancer $(67.4 \%)$ and gynecological cancer types, including ovarian cancer $(61.5 \%)$ and endometrial cancer $(57.3 \%)$. Compared with that in the respective normal tissues, ING2 expression in breast cancer tissues was decreased, while that in cervical cancer was upregulated in the nuclei as well as the cytoplasm. In endometrial cancer, expression of ING2 was increased in the nuclei and declined in the cytoplasm compared with that in the normal endometrium. ING2-positive cases were less frequent for renal clear cell carcinoma (17.7\%). The results of the present study suggested that ING2 may be involved in the repair and regeneration of organs or tissues and is associated with breast and gynecological carcinogenesis.

\section{Introduction}

The gene encoding inhibitor of growth protein 2 (ING2) is made up of three exons (1a, $1 \mathrm{~b}$ and 2) and encodes two transcript isoforms (ING2a and ING2b) by alternative splicing. The ING2 C-terminal domain contains a plant homeodomain (PHD) with a zinc-binding motif, which has a high affinity for the histone 3 tri-methylated on lysine 4 and a nuclear localization sequence (NLS) (1). Initially, ING2a was shown to regulate cell proliferation, cell-cycle arrest, senescence and apoptosis due to enhancing p53 transcription and acetylation on lysine 382 by interaction with p300 acetyltransferase, which promotes p53 target genes (eg. p21 and $\mathrm{Bcl}-2$-associated $\mathrm{X}$ protein) (2-4). In response to exogenous stresses, the p38 kinase pathway is activated to regulate phosphorylation of phosphatidylinositol 5-phosphate, resulting in an induction of ING2 accumulation to the chromatin and subsequent modulation of p53 acetylation and ING2 targets (3-7). ING2 protein recruits and stabilizes the $\mathrm{mSin} 3 \mathrm{a} /$ higherhistonedeacetylase 1 (HDAC)1-2/Sin3a-associated protein complex, and regulates target gene expression (1), which has been enhanced by phosphorylation-dependent ING2a sumoylation by small ubiquitin-like modifier 1 on lysine 195 (8), while ubiquitin ligase SMAD-specific E3 ubiquitin protein ligase 1 targets the tumor suppressor ING2 for ubiquitination and degradation (9). In addition, ING2a binds to Ski-like oncogene (SnoN), a SMAD-interacting transcriptional modulator, through its PHD domain to finally 
suppress cell proliferation, suggesting that ING2 collaborates with SnoN to mediate transforming growth factor $\beta$-induced SMAD-dependent transcription and cellular responses (10).

ING2a mRNA is highly expressed in skeletal muscle, lung, thymus, pancreas and testis $(11,12)$. Although human ING2a and $2 \mathrm{~b}$ have a molecular weight of 33 and $28 \mathrm{kDa}$, respectively, ING2b protein has never been experimentally detected $(1,12,13)$. Saito et al (12) have reported that ING2-/mice develop soft-tissue sarcomas with an incidence of $46 \%$, among which histiocytic sarcomas were most frequent (28\%). The ING2-/- male mice appeared infertile because of deficient spermatogenesis, small testes, seminiferous tubule degeneration, abnormal spermatozoal motility and morphology from the age of eight weeks. A high frequency of loss of heterozygosity (LOH) of the ING2 chromosomal region 4q32-35.1 has been found in basal cell carcinomas (14), head and neck squamous cell carcinomas $(15,16)$, and hepatocellular carcinomas (HCC) (17). LOH of ING2 is associated with advanced tumor stages in head and neck squamous cell carcinomas (15). ING2 expression is downregulated in various cancer types, including melanoma and HCC (17), non-small cell lung carcinomas (18) and melanoma (19). The expression levels of ING2 protein were negatively correlated with tumor size, histopathological classification and serum alpha-fetoprotein in HCC, but may be employed as an independent prognostic factor to indicate favorable prognosis (17). By contrast, Kumamoto et al (20) demonstrated that ING2 mRNA overexpression was associated with colon carcinogenesis by upregulating the expression of matrix metalloproteinase 13 via the formation of an ING2-HDAC1-mSin3A complex.

Identification of normal tissues or cell types which express ING2 may contribute towards clarifying the physiological function of ING2, while the elucidation of its expression pattern as well as its heterogeneity among tumor cases or between tumor and normal tissues may lead to its identification as a target for gene therapy, which may be evaluated using animal models of conditional ING2 knockout (1). A previous study employed intermittent microwave irradiation for immunohistochemical analysis of ING2, during which $>2.4$ billion vibrations per minute enhanced the probability of specific antibody-antigen recognition (21). The present study adopted this technique to perform immunohistochemical expression profiling of ING2 protein in normal mouse and human tissues as well as in human cancer tissues.

\section{Materials and methods}

Specimens. Three male and three female C57BL/6 mice (eight weeks old; $25 \mathrm{~g}$ ) were sacrificed under sodium pentobarbital anesthesia and brain, heart, liver, spleen, lung, kidney, breast, stomach and intestinal tissue samples were excised. All tissues were fixed in $10 \%$ neutral formalin and embedded in paraffin. An array of normal human tissues (cerebrum, cerebellum, brain stem, aorta, tongue, thyroid, esophagus, stomach, intestine, liver, pancreas, lung, trachea, appendix, smooth, muscle, skeletal muscle, heart, testis, bladder and prostate) and cancer tissues (62 hepatocellular carcinomas, 62 renal clear cell carcinomas, 62 pancreatic carcinomas, 45 esophageal squamous cell carcinomas and 31 cervical squamous cell carcinomas) were purchased from Shanghai Outdo Biotech
Co., Ltd. (Shanghai, China). Normal human cervical, endometrial, ovary and breast tissues, as well as breast $(n=144)$, gastric $(n=196)$, colorectal $(n=96)$, ovarian $(n=208)$, endometrial $(n=96)$ and lung carcinoma $(n=192)$ tissues were obtained at The First Affiliated Hospital of Liaoning Medical University (Jinzhou, China). The normal mouse and human tissues, and the cancer tissues were also subjected to a tissue microarray using a tissue microarray apparatus (KIN-1; Azumaya, Tokyo, Japan). No patients with cancer had undergone chemotherapy, radiotherapy, or adjuvant treatment prior to surgery. The patients or their relatives provided written consent for the use of tumor tissues for clinical research, and the research protocol was approved by the Ethical and Animal Experimentation Committees of Liaoning Medical University (Jinzhou, China).

Immunohistochemistry. Consecutive sections were de-waxed with xylene, debenzolization with ethanol in water and subjected to antigen retrieval by irradiation in target retrieval solution (DAKO, Glostrup, Denmark) in a microwave oven for 15 min (500 W; Oriental Rotor Ltd Co., Tokyo, Japan). Sections were then blocked in $5 \%$ bovine serum albumin (Shanghai Chemical Technology Co., Ltd., Shanghai, China) for $5 \mathrm{~min}$ to prevent non-specific antibody binding. The sections were incubated with rabbit anti-ING2 antibody (cat no. 11560-1-AP; Proteintech, Chicago, IL USA; 1:50 dilution) for $15 \mathrm{~min}$, followed by incubation with anti-rabbit secondary antibody conjugated to horseradish peroxidase (DAKO) for $15 \mathrm{~min}$. All of the incubations were cross-linked in a microwave oven at $37^{\circ} \mathrm{C}$ in order to allow intermittent irradiation as previously described (21). After each treatment, the slides were washed with Tris-buffered saline containing Tween 20 (3x1 min). Bound antibodies were then stained using 3,3'-diaminobenzidine (Sangon Biotech Co., Ltd., Shanghai, China). After counterstaining with Mayer's hematoxylin, the sections were de-hydrated, cleared and mounted. Normal mouse immunoglobulin G (Santa Cruz Biotechnology, Inc., Santa Cruz, CA, USA) was used instead of the primary antibody as a negative control.

Immunostaining evaluation. As indicated in Figs. 1-3, ING2 protein was localized to the cytoplasm or/and nuclei. Initially, the high-expression field was selected at low magnification and one hundred cells were randomly counted from five different representative fields at a high magnification by two independent examiners (Miss S Zhao and Professor HC Zheng). In the case of conflicting data, the two examiners continued until a final agreement was reached. The percentages of counted cells were scored as follows: $0-10 \%$, negative (-); and 11-100\%, positive (+). The density of immunostaining was assessed using ImagePro Plus software, version 16.0 (Media Cybernetics, Rockville, MD, USA). The percentage of ING2-positive cells was graded semi-quantitatively according to a four-tier scoring system: (-), negative; (+), weakly positive; (++) moderately positive; and $(+++)$ strongly positive.

\section{Results}

As shown in Fig. 1, the sub-cellular location of ING2 was in the nuclei, cytoplasm or nucleocytoplasm in the mouse tissues in either sporadic or localized patterns, although expression levels 
Table I. Immunohistochemical detection of inhibitor of growth protein 2 expression in normal mouse tissues.

\begin{tabular}{lll}
\hline Tissue source & \multicolumn{1}{c}{ Tissue type } & Localization \\
\hline Brain & & Scattered \\
Heart & Myocardial fibroblasts & Nuclei and cytoplasm \\
Lung & Bronchial glandular epithelial cells & Nuclei and cytoplasm \\
& Alveolar glandular epithelial cells & Nuclei and cytoplasm \\
Kidney & Renal tubules & Cytoplasm \\
& Glomeruli & Nuclei and cytoplasm \\
Intestine & Glandular epithelium & Nuclei and cytoplasm \\
Spleen endothelium & & Scattered \\
Skin & Squamous epithelium & Nuclei and cytoplasm \\
Striated muscle & Striated muscle cells & Cytoplasm \\
Liver & Glandular epithelium & Nuclei and cytoplasm \\
Breast & Glandular epithelium & Nuclei and cytoplasm \\
\end{tabular}

Table II. Immunohistochemical detection of ING2 expression and localization in normal human tissues.

\begin{tabular}{|c|c|c|}
\hline \multirow[b]{2}{*}{ Tissue type } & \multicolumn{2}{|c|}{ ING2 expression } \\
\hline & Nucleus & Cytoplasm \\
\hline Cerebrum & - & ++ \\
\hline Cerebellum & - & ++ \\
\hline Brain stem & - & + \\
\hline Thymus & - & + \\
\hline Skeletal muscle & - & ++ \\
\hline Aorta & - & ++ \\
\hline Tongue & +++ & +++ \\
\hline Thyroid & - & + \\
\hline Esophagus & ++ & ++ \\
\hline Stomach & +++ & ++ \\
\hline Intestine & ++ & ++ \\
\hline Liver & - & ++ \\
\hline Pancreas & + & +++ \\
\hline Lung & - & + \\
\hline Trachea & - & ++ \\
\hline Skin & ++ & +++ \\
\hline Appendix & + & ++ \\
\hline Muscle & - & + \\
\hline Striated muscle & - & + \\
\hline Smooth muscle & - & ++ \\
\hline Testis & - & + \\
\hline Bladder & - & + \\
\hline Prostate & - & ++ \\
\hline Cervix & ++ & +++ \\
\hline Endometrium & +++ & ++ \\
\hline Ovary & - & + \\
\hline Breast & +++ & ++ \\
\hline
\end{tabular}

Scoring system: (-), negative; (+), weakly positive, $(++)$, moderately positive; $(+++)$, strongly positive. ING2, inhibitor of growth protein 2 .

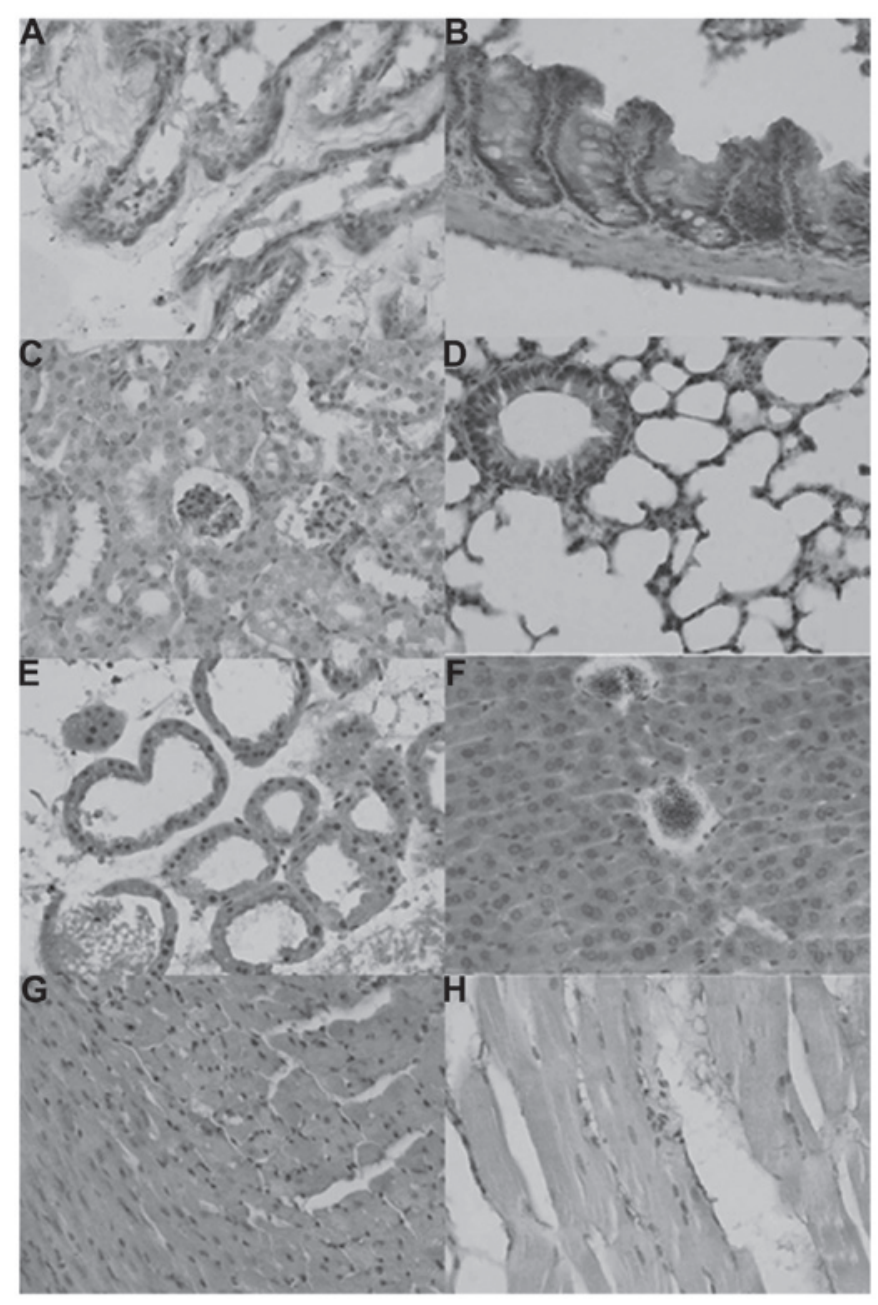

Figure 1. Immunohistochemical detection of inhibitor of growth protein 2 expression in normal mouse tissues. (A) Skin; (B) intestine; (C) kidney; (D) lung and trachea; (E) breast; (F) liver; (G) myocardium; (H) striated muscle.

differed among tissues and cell populations. ING2 reactivity was detectable in the nuclei as well as the cytoplasm of the glandular epithelium of the breast, liver, intestine, bronchium 


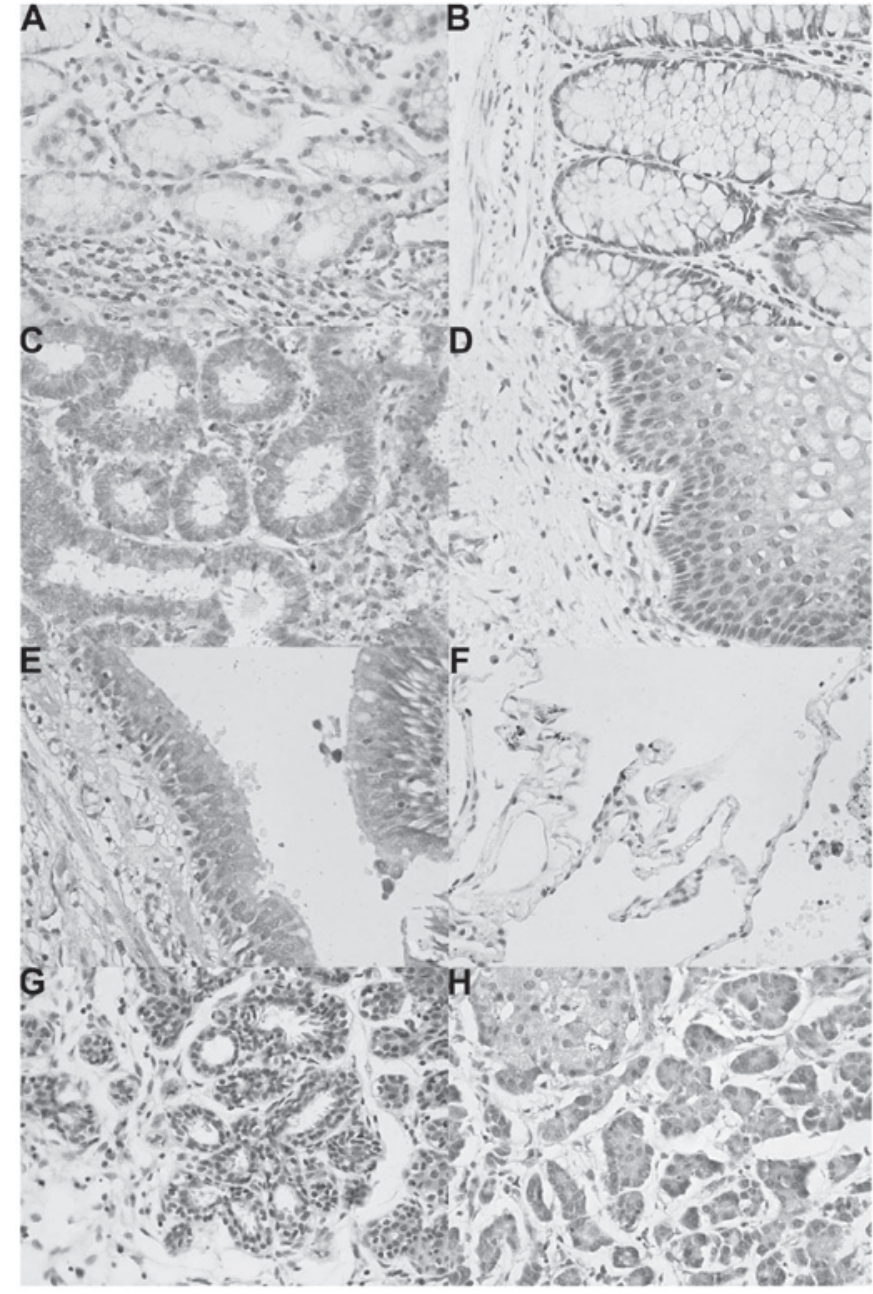

Figure 2. Immunohistochemical detection of inhibitor of growth protein 2 expression and localization in normal human tissues. (A) Stomach (B) intestine; (C) endometrium; (D) cervix; (E) trachea; (F) lung; (G) breast; (H) pancreas.

and alveolum, the squamous epithelium of the skin, glomeruli, and in myocardial cells, while it was located in the cytoplasm of renal tubules and striated muscle cells. ING2 protein was scattered in the brain and spleen (Table I).

In human tissues, ING2 protein was primarily distributed in the cytoplasm; however, it was located in the cytoplasm and nuclei of tissues from the stomach, intestine, cervix, trachea, breast and pancreas. In the stomach, the nuclear location of ING2 was more prominent than that in the cytoplasm (Fig. 2). According to the density of the immunostaining, ING2 was highly expressed in the tongue, stomach, skin, pancreas, cervix and breast, whereas it was weakly expressed in the brain stem, thymus, thyroid, lung, striated muscle, testis, bladder and ovary (Table II).

In total, 617 out of 1,194 cancer tissues assessed in the present study were ING2-positive (51.7\%), with a homogeneity in their expression pattern (Fig. 3; Table III). ING2 expression was detected in the cytoplasm of all cancer types, whereas it was present in the nuclei of certain cancer tissues, including lung, breast and endometrial cancers. Among them, ING2 was more frequently expressed in breast cancer $(67.4 \%$; 97/144) and gynecological cancers, including ovarian cancer $(61.5 \%$; $128 / 208)$ and endometrial cancer $(57.3 \% ; 55 / 96)$. Compared

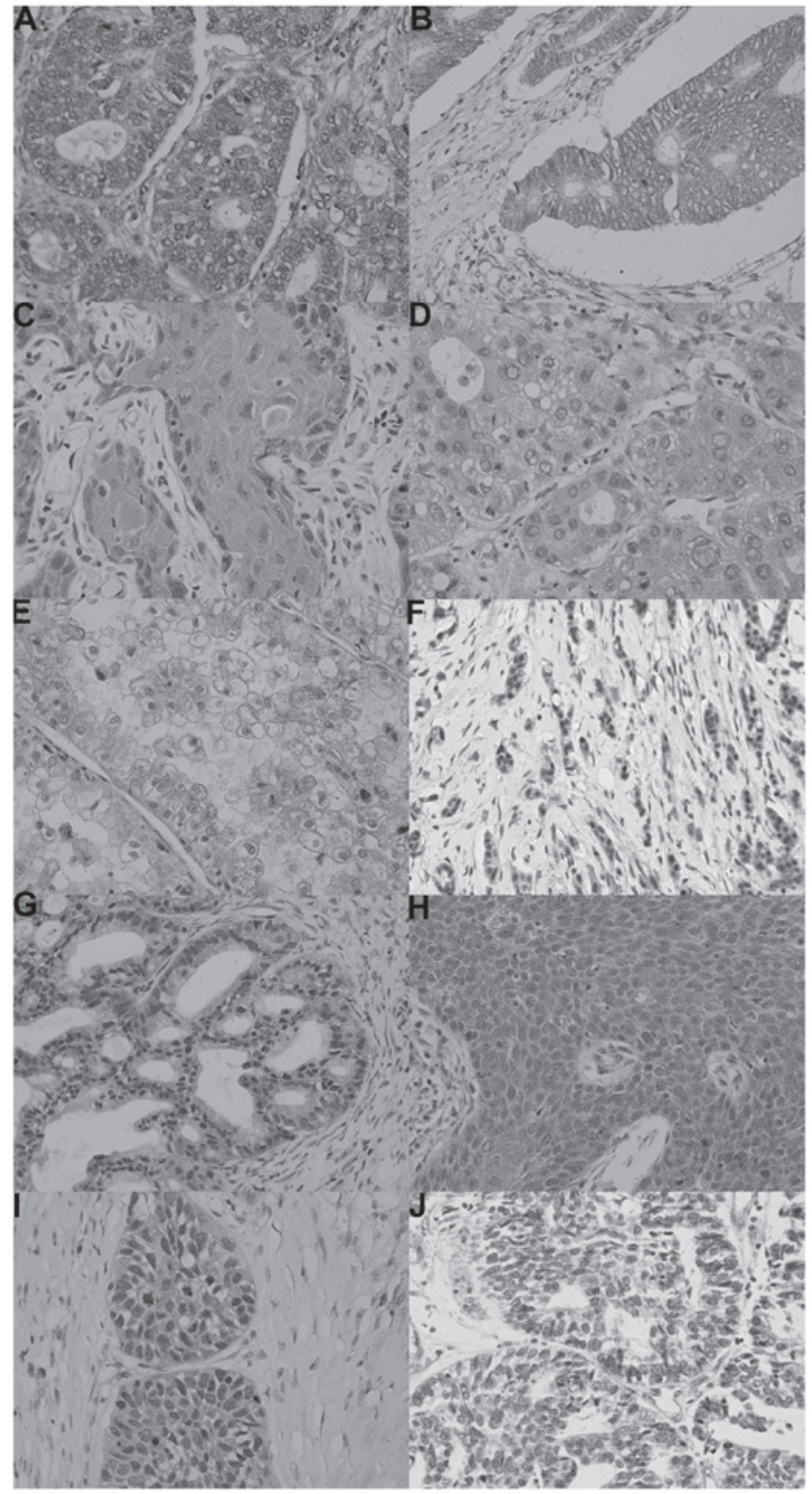

Figure 3. Immunohistochemical detection of inhibitor of growth protein 2 expression and localization in human cancers. (A) Gastric carcinoma; (B) colorectal cancer; (C) esophageal carcinoma; (D) hepatocellular carcinoma; (E) renal clear cell carcinoma; (F) breast cancer; $(\mathrm{G})$ ovarian cancer; (H) cervical carcinoma; (I) lung carcinoma; (J) endometrial carcinoma.

with their respective normal tissues, ING2 expression in breast cancer tissues was decreased, while it was upregulated in the nuclei as well as in the cytoplasm of cervical cancer tissues, whereas ING2 was increased in the nuclei and declined in the cytoplasm of endometrial cancer tissues (Fig. 4). By contrast ING2-positive cases were less frequent in renal clear cell carcinoma $(17.7 \% ; 11 / 62)$.

\section{Discussion}

ING2 has been identified and characterized as a Type-II tumor suppressor gene as its involvement in cancer appears to be linked to its level of expression rather than its mutational status $(1,18)$. ING2 protein contains NLS and PHD 
Table III. Immunohistochemical detection of ING2 expression in human cancer tissues.

ING2 expression

\begin{tabular}{|c|c|c|c|c|c|}
\hline \multirow[b]{2}{*}{ Cancer type } & \multirow[b]{2}{*}{$\mathrm{n}$} & \multirow[b]{2}{*}{ Positive cases (n) } & \multirow[b]{2}{*}{ PR $(\%)$} & \\
\hline & & & & Nucleus & Cytoplasm \\
\hline Hepatocellular carcinoma & 62 & 25 & 40.3 & - & + \\
\hline Renal cell carcinoma & 62 & 11 & 17.7 & - & + \\
\hline Pancreatic cancer & 62 & 20 & 32.3 & - & + \\
\hline Esophageal carcinoma & 45 & 19 & 42.2 & + & + \\
\hline Cervical carcinoma & 31 & 15 & 48.4 & + & + \\
\hline Breast cancer & 144 & 97 & 67.4 & + & + \\
\hline Gastric carcinoma & 196 & 85 & 43.4 & + & + \\
\hline Colorectal carcinoma & 96 & 56 & 58.3 & + & + \\
\hline Ovarian cancer & 208 & 128 & 61.5 & + & + \\
\hline Endometrial carcinoma & 96 & 55 & 57.3 & + & + \\
\hline Lung carcinoma & 192 & 106 & 55.2 & + & + \\
\hline
\end{tabular}

Scoring system: (-), negative, 0-10\%; (+), positive, 11-100\%. ING2, inhibitor of growth protein 2; PR, positive rate.

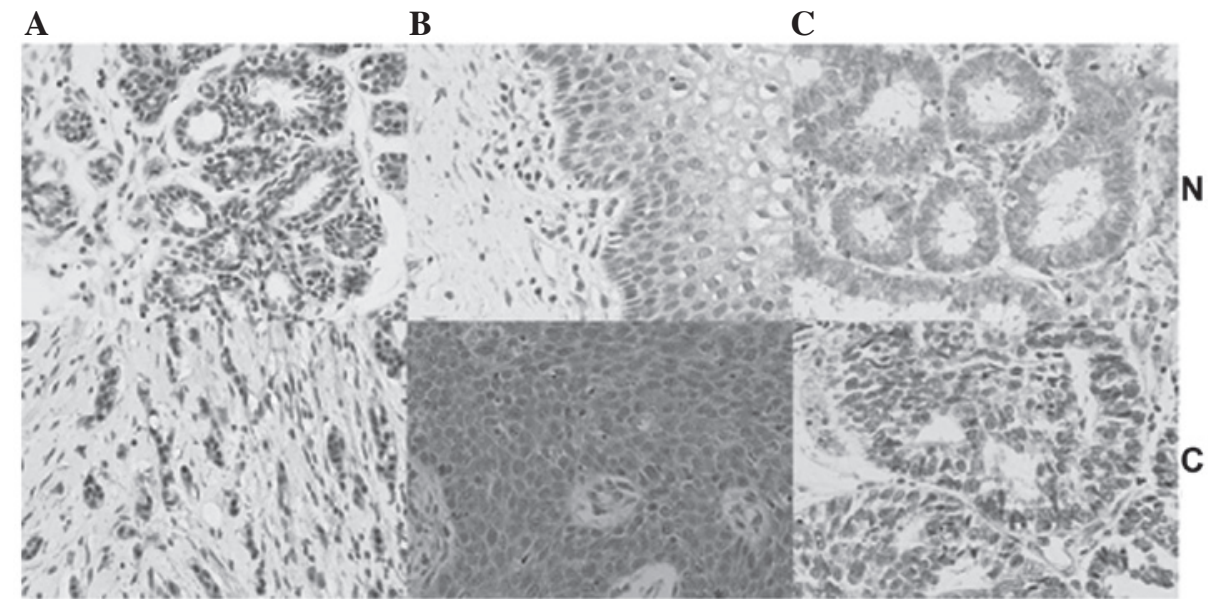

Figure 4. Immunohistochemical detection of inhibitor of growth protein 2 expression and localization in human cancers. (A) Breast; (B) cervix; (C) endometrium. $\mathrm{N}$, normal tissue; $\mathrm{C}$, cancer tissue.

finger motifs in its C-terminus (1). In the present study, the expression levels and cellular localization of ING2 protein were determined in a tissue array comprising normal mouse and human tissues, as well as human cancer tissues. Positive expression of ING2 was observed in the cytoplasm of normal mouse and human tissues as well as human cancer tissues; however, ING2 was present in the cytoplasm as well as in the nuclei in certain tissue types. It has been reported that $\mathrm{LOH}$ of the ING2 chromosomal region or the ING2 gene may result in the downregulation of its expression (14-17). Cenzig et al (22) found that the mutation or deletion of ING5 NLS is responsible for the nucleocytoplasmic translocation. Zhang et al (17) demonstrated that immunostaining for ING2 was mostly located to the cytoplasm, while weak nuclear staining was also observed in HCC tissues and normal hepatocytes, in line with a study by Gozani et al (5), who reported that phosphatidylinositol 5-phosphate 4-kinase type II $\beta$ expression decreased endogenous nuclear ING2 in HT1080 fibrosarcoma cells. It has been reported that ING1

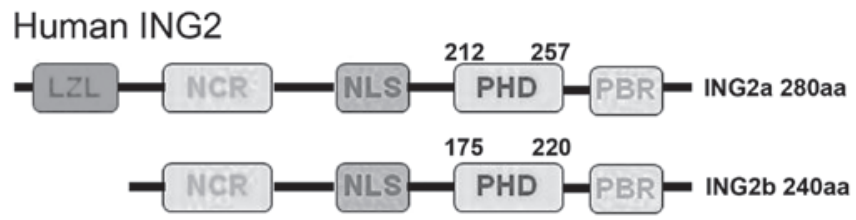

Mouse ING2

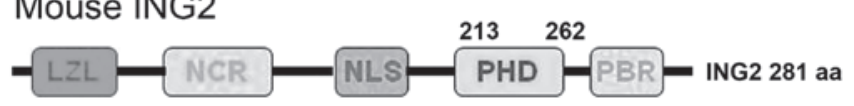

Figure 5. Schematic diagram of human and mouse ING2 protein structure. ING2, inhibitor of growth protein 2; LZL, leucine zipper-like motif; NCR, novel conserved region; NLS, nuclear location sequence; PHD, plant homeodomain; PBR, poly basic region.

phosphorylation by 14-3-3 family members (23) or Src (24) caused its cytoplasmic re-localization leading to apoptotic induction. Future studies should investigate the purpose of ING2 restoration in the cytoplasm as well as its function. 
Amino acid sequence alignment has revealed a high similarity between human ING2a and mouse ING2 (96\% identity) (Fig. 5) (1); this may explain for the findings of the present study, which revealed no substantial differences in the patterns of ING2 expression between mouse and human samples, with the exception of sub-cellular location in certain cell types. In human tissues, high expression of ING2 protein was detected in the tongue, stomach, skin, pancreas, cervix and breast, while its expression was low in the brain stem, thymus, thyroid, lung, striated muscle, testis, bladder and ovary, suggesting the functional involvement of ING2 in the functions of specific cell types or in cells in a specific functional state. In order to further investigate the roles of ING2, our group has inhibited ING2 using a cell-specific promoter in order to establish an animal model of ING2-negative cancer. Previous studies have highlighted the anti-proliferative and apoptotic functions of ING2 $(4,8)$. ING2 overexpression in the stomach, tongue, skin, pancreas, cervix and breast may indicate its association with regenerative processes regardless of the identity of the cells being glandular or squamous epithelium, which is supported by the finding of relatively low expression in organs with a decreased capacity for cell repair and renewal, including the brain stem, thymus, thyroid, skeletal muscle and testis. Of note, ING2 has been involved in muscle differentiation by regulating myogenin transcription (25), providing an explanation for ING2 overexpression in muscle cells.

ING2 is a candidate tumor suppressor gene, whose expression is frequently lost in tumors. The present study focused on common epithelial cell-derived tumors and demonstrated that breast cancer and gynecological cancer types, including ovarian and endometrial carcinoma, showed a high incidence of ING2 expression, indicating that ING2 protein may be closely linked to estrogen production. By contrast, the positive rate for ING2 expression in renal clear cell carcinoma was $<20 \%$. This finding may indicate that ING2 may be used as a therapeutic target in renal clear cell carcinoma. ING2 was reported to interact with proliferating cell nuclear antigen and regulates its quantity of the chromatin fraction, thereby directly maintaining DNA replication and integrity $(26,27)$. The ING2 C-terminus recruits histone methyltransferase activity and trimethylates histone $\mathrm{H} 3$ at lysine 9 (28). The leucine zipper-like motif of ING2 is critical for the proper functioning of ING2 in DNA repair, apoptosis and p53-dependent chromatin remodeling by acting as a scaffold protein to mediate the interaction between p53 and p300 (3-7). The profiling of ING2 expression performed in the present study provided useful hints regarding the disruption of DNA replication, proliferation and apoptosis in various epithelial cancer types.

In conclusion, the present study not only elucidated the abundant expression of ING2 in normal mouse and human tissues as well as human cancer tissues, but also demonstrated the differential expression and/or sub-cellular location of ING2 among various tissues, cell types and single cells, suggesting differential functional involvement. According to the results of the present study, it is hypothesized that ING2 may be involved in the repair and regeneration of organs or tissues and have an important role in gynecological carcinogenesis.

\section{Acknowledgements}

The present study was supported by the Natural Science Foundation of Liaoning Province (no. 2013022070) and the National Natural Scientific Foundation of China (no. 81172371).

\section{References}

1. Guérillon C, Larrieu D and Pedeux R: ING1 and ING2: Multifaceted tumor suppressor genes. Cell Mol Life Sci 70: 3753-3772, 2013

2. Pedeux R, Sengupta S, Shen JC, Demidov ON, Saito S, Onogi H, Kumamoto K, Wincovitch S, Garfield SH, McMenamin M, et al: ING2 regulates the onset of replicative senescence by induction of p300-dependent p53 acetylation. Mol Cell Biol 25: 6639-6648, 2005.

3. Bua DJ, Martin GM, Binda O and Gozani O: Nuclear phosphatidylinositol-5-phosphate regulates ING2 stability at discrete chromatin targets in response to DNA damage. Sci Rep 3: 2137, 2013.

4. Larrieu D, Ythier D, Brambilla C and Pedeux R: ING2 controls the G1 to S-phase transition by regulating p21 expression. Cell Cycle 9: 3984-3990, 2010.

5. Gozani O, Karuman P, Jones DR, Ivanov D, Cha J, Lugovskoy AA, Baird CL, Zhu H, Field SJ, Lessnick SL, et al: The PHD finger of the chromatin-associated protein ING2 functions as a nuclear phosphoinositide receptor. Cell 114: 99-111, 2003.

6. Huang W, Zhang H, Davrazou F, Kutateladze TG, Shi X, Gozani O and Prestwich GD: Stabilized phosphatidylinositol-5-phosphate analogues as ligands for the nuclear protein ING2: Chemistry, biology and molecular modeling. J Am Chem Soc 129: 6498-6506, 2007.

7. Jones DR, Bultsma Y, Keune WJ, Halstead JR, Elouarrat D, Mohammed S, Heck AJ, D'Santos CS and Divecha N: Nuclear PtdIns5P as a transducer of stress signaling: An in vivo role for PIP4Kbeta. Mol Cell 23: 685-695, 2006.

8. Ythier D, Larrieu D, Binet R, Binda O, Brambilla C, Gazzeri S and Pedeux R: Sumoylation of ING2 regulates the transcription mediated by Sin3A. Oncogene 29: 5946-5956, 2010.

9. Nie J, Liu L, Wu M, Xing G, He S, Yin Y, Tian C, He F and Zhang L: HECT ubiquitin ligase smurf1 targets the tumor suppressor ING2 for ubiquitination and degradation. FEBS Lett 584: 3005-3012, 2010.

10. Sarker KP, Kataoka H, Chan A, Netherton SJ, Pot I, Huynh MA, Feng X, Bonni A, Riabowol K and Bonni S: ING2 as a novel mediator of transforming growth factor-beta-dependent responses in epithelial cells. J Biol Chem 283: 13269-13279, 2008.

11. Walzak AA, Veldhoen N, Feng X, Riabowol K and Helbing CC: Expression profiles of mRNA transcript variants encoding the human inhibitor of growth tumor suppressor gene family in normal and neoplastic tissues. Exp Cell Res 314: 273-285, 2008.

12. Saito M, Kumamoto K, Robles AI, Horikawa I, Furusato B, Okamura S, Goto A, Yamashita T, Nagashima M, Lee TL, et al: Targeted disruption of Ing2 results in defective spermatogenesis and development of soft-tissue sarcomas. PLoS One 5: e15541, 2010.

13. Nagashima M, Shiseki M, Miura K, Hagiwara K, Linke SP, Pedeux R, Wang XW, Yokota J, Riabowol K and Harris CC: DNA damage-inducible gene p33ING2 negatively regulates cell proliferation through acetylation of $\mathrm{p} 53$. Proc Natl Acad Sci USA 98: 9671-9676, 2001.

14. Sironi E, Cerri A, Tomasini D, Sirchia SM, Porta G, Rossella F, Grati FR and Simoni G: Loss of heterozygosity on chromosome 4q32-35 in sporadic basal cell carcinomas: Evidence for the involvement of p33ING2/ING1L and SAP30 genes. J Cutan Pathol 31: 318-322, 2004.

15. Borkosky SS, Gunduz M, Nagatsuka H, Beder LB, Gunduz E, Ali MA, Rodriguez AP, Cilek MZ, Tominaga S, Yamanaka N, et al: Frequent deletion of ING2 locus at 4q35.1 associates with advanced tumor stage in head and neck squamous cell carcinoma. J Cancer Res Clin Oncol 135: 703-713, 2009. 
16. Cetin E, Cengiz B, Gunduz E, Gunduz M, Nagatsuka $\mathrm{H}$, Bekir-Beder L, Fukushima K, Pehlivan D, N MO, Nishizaki K, et al: Deletion mapping of chromosome 4q22-35 and identification of four frequently deleted regions in head and neck cancers. Neoplasma 55: 299-304, 2008.

17. Zhang HK, Pan K, Wang H, Weng DS, Song HF, Zhou J, Huang W, Li JJ, Chen MS and Xia JC: Decreased expression of ING2 gene and its clinicopathological significance in hepatocellular carcinoma. Cancer Lett 261: 183-192, 2008.

18. Ythier D, Brambilla E, Binet R, Nissou D, Vesin A, de Fraipont F, Moro-Sibilot D, Lantuejoul S, Brambilla C, Gazzeri S and Pedeux R: Expression of candidate tumor suppressor gene ING2 is lost in non-small cell lung carcinoma. Lung Cancer 69 : 180-186, 2010.

19. Lu F, Dai DL, Martinka M, Ho V and Li G: Nuclear ING2 expression is reduced in human cutaneous melanomas. Br J Cancer 95: 80-86, 2006.

20. Kumamoto K, Fujita K, Kurotani R, Saito M, Unoki M, Hagiwara N, Shiga H, Bowman ED, Yanaihara N, Okamura S, et al: ING2 is upregulated in colon cancer and increases invasion by enhanced MMP13 expression. Int J Cancer 125: 1306-1315, 2009.

21. Kumada T, Tsuneyama K, Hatta H, Ishizawa S and Takano Y: Improved 1-h rapid immunostaining method using intermittent microwave irradiation: Practicability based on 5 years application in Toyama medical and pharmaceutical university hospital. Mod Pathol 17: 1141-1149, 2004.
22. Cengiz BI, Gunduz E, Gunduz M, Beder LB, Tamamura R, Bagci C, Yamanaka N, Shimizu K and Nagatsuka H: Tumor-specific mutation and downregulation of ING5 detected in oral squamous cell carcinoma. Int J Cancer 127: 2088-2094, 2010.

23. Gong W, Russell M, Suzuki K and Riabowol K: Subcellular targeting of p33ING1b by phosphorylation-dependent 14-3-3 binding regulates p21WAF1 expression. Mol Cell Biol 26: 2947-2954, 2006

24. Yu L, Thakur S, Leong-Quong RY, Suzuki K, Pang A, Bjorge JD, Riabowol K and Fujita DJ: Src regulates the activity of the ING1 tumor suppressor. PLoS One 8: e60943, 2013.

25. Eapen SA, Netherton SJ, Sarker KP, Deng L, Chan A, Riabowol K and Bonni S: Identification of a novel function for the chromatin remodeling protein ING2 in muscle differentiation. PLoS One 7: e40684, 2012.

26. Larrieu D, Ythier D, Binet R, Brambilla C, Brambilla E, Sengupta S and Pedeux R: ING2 controls the progression of DNA replication forks to maintain genome stability. EMBO Rep 10: 1168-1174, 2009.

27. Goeman F, Otto K, Kyrylenko S, Schmidt O and Baniahmad A: ING2 recruits histone methyltransferase activity with methylation site specificity distinct from histone $\mathrm{H} 3$ lysines 4 and 9 . Biochim Biophys Acta 1783: 1673-1680, 2008.

28. Wang Y, Wang J and Li G: Leucine zipper-like domain is required for tumor suppressor ING2-mediated nucleotide excision repair and apoptosis. FEBS Lett 580: 3787-3793, 2006. 